\title{
Measuring the sensitivity to optical feedback of single-frequency high-power laser
} diodes

Christensen, Mathias; Zink, Christof; Jamal, Muhammad Tahir; Hansen, Anders Kragh; Jensen, Ole Bjarlin; Sumpf, Bernd

Published in:

Journal of the Optical Society of America B-optical Physics

Link to article, DOI:

10.1364/JOSAB. 417258

Publication date:

2021

Document Version

Peer reviewed version

Link back to DTU Orbit

Citation (APA):

Christensen, M., Zink, C., Jamal, M. T., Hansen, A. K., Jensen, O. B., \& Sumpf, B. (2021). Measuring the sensitivity to optical feedback of single-frequency high-power laser diodes. Journal of the Optical Society of America B-optical Physics, 38(3), 885-892. https://doi.org/10.1364/JOSAB.417258

\section{General rights}

Copyright and moral rights for the publications made accessible in the public portal are retained by the authors and/or other copyright owners and it is a condition of accessing publications that users recognise and abide by the legal requirements associated with these rights.

- Users may download and print one copy of any publication from the public portal for the purpose of private study or research.

- You may not further distribute the material or use it for any profit-making activity or commercial gain

- You may freely distribute the URL identifying the publication in the public portal 


\title{
Measuring the sensitivity to optical feedback of single-frequency high-power laser diodes
}

\author{
MAthias Christensen ${ }^{1,2}$, ChRistof ZinK ${ }^{3}$, Muhammad TAHIR JAMAL ${ }^{4}$, \\ Anders Kragh Hansen ${ }^{1}$, Ole Bjarlin Jensen ${ }^{1, *}$, Bernd SumpF ${ }^{3}$ \\ ${ }^{I}$ Technical University of Denmark, Diode Lasers and LED Systems, Frederiksborgvej 399, 4000 \\ Roskilde Denmark \\ ${ }^{2}$ Currently with Norlase ApS, Brydehusvej 13, 2750 Ballerup Denmark \\ ${ }^{3}$ Ferdinand-Braun-Institut, Leibniz-Institut für Höchstfrequenztechnik, Berlin 12489, Germany \\ ${ }^{4}$ DTU Health Tech, Technical University of Denmark, Frederiksborgvej 399, 4000 Roskilde, Denmark \\ *ojen@fotonik.dtu.dk
}

\begin{abstract}
Laser diodes, in general, are sensitive to optical feedback, especially with regards to maintaining single-frequency operation. Until now, however, the feedback sensitivity of high-power devices such as single-frequency DBR tapered laser diodes has not been investigated in quantitative detail. In this paper, we analyze the impact of very weak optical feedback between $-105 \mathrm{~dB}$ and $-40 \mathrm{~dB}$ on a high-power DBR tapered laser diode. The measurement set-up is validated using a typical DFB laser diode. The results are in good agreement with theory at low feedback levels.
\end{abstract}

(c) 2020 Optical Society of America under the terms of the OSA Open Access Publishing Agreement

\section{Introduction}

For most applications, an ideal laser source would be a semiconductor-based laser diode due to the high efficiency, small size, and low cost when produced in large quantities. In recent years, developments in tapered laser diodes have proved that it is possible to manufacture laser diodes in the near infrared emitting more than $10 \mathrm{~W}$ of optical power at a single frequency and with the majority of the power spatially contained in a Gaussian-like central lobe [1-3]. With electro-optical efficiencies around $40-50 \%$ and a size of a few $\mathrm{mm}^{3}$, these devices are ideal candidates for second harmonic generation (SHG) into the visible spectral range [4] or remote sensing applications [5]. However, reliable single-frequency operation in a system incorporating such high-power single-frequency diode lasers often requires optical isolation between the optical elements and the laser itself between 30 and $60 \mathrm{~dB}$ due to the risk of spectral instability or even laser degradation. Unfortunately, the Faraday isolators necessary to achieve this isolation at $10 \mathrm{~W}$ of power are bulky, expensive, and use strong magnetic fields to achieve high isolation. All of these parameters can counteract some of the benefits offered by the laser diodes.

While external cavity diode lasers, obtained with high optical feedback power ratios, have been studied extensively [6,7], the opposite is true for low levels of feedback, which are sometimes known as parasitic reflections. The majority of the work at low feedback power ratios was done during the early telecom years and has revolved around avoiding a state known as coherence collapse for distributed feedback (DFB) lasers often caused by reflections from fiber couplings $[8,9]$. However, the first effects of the feedback can be seen at feedback levels, which are two or three orders of magnitude lower than the levels required for coherence collapse. These smaller effects are often acceptable for communication systems where short devices with a large mode spacing are used. However, for the smaller mode spacing of the relatively long wavelength stabilized high-power lasers, e.g., tapered laser diodes, small frequency perturbations from feedback can lead to mode hopping. This mode hopping can be detrimental, e.g., SHG, where a wavelength shift of tens of picometers can significantly change the conversion efficiency [10]. Some work has also concerned a more general 
description of the different effects seen at low feedback power ratios, both experimentally [11] and numerically $[12,13]$. The use of these feedback effects in sensitive measurements using self-mixing interferometry is described in [14]. Here, a good overview of the background and a collection of application examples are given showing the potential of the technique. While the focus of the experimental work was mainly on ridge-waveguide DFB laser diodes, the theory can be applied to any laser emitting in a single longitudinal mode. Recent work has also shown that semiconductor ring lasers are less sensitive to optical feedback [15]. Unfortunately, the measurement setups and methods described in the literature are not suitable for quantitative characterization of the feedback sensitivity of single-frequency high power laser diodes such as distributed Bragg reflector tapered laser diodes (DBR-TPL). This is because they either rely on feedback to the back facet of the device, which is not a realistic case for DBR tapered laser diodes, or they are already very specific for only one application, e.g., feedback from a single-mode fiber [16] or a SHG crystal [17]. Overall, the existing literature gives little insight into the challenges associated with designing and using a setup where the results can be impacted by feedback power ratios as low as $-80 \mathrm{~dB}$, nor does it describe the reproducibility of the results.

In this paper, we give a detailed description of a measurement method to determine the sensitivity to external feedback from a relatively long distance of high-power frequency stabilized laser diodes. This is particularly relevant when using such laser sources for nonlinear frequency conversion. The measurement method was tested with a low power ridgewaveguide DFB laser and later on applied to a high power DBR-TPL. The experimental results of the measurements are discussed and compared to simulations based on the LangKobayashi equations.

\section{Theoretical background}

The most widely used theory to describe optical feedback to laser systems is based on the Lang-Kobayashi equations [18]. These equations are valid for a laser emitting in a single longitudinal mode.

In the literature, the spectral effects of feedback have previously been divided into 5 regimes [11]. Regime I is defined by any feedback level at which only one steady-state solution exists for the laser at all phases of the feedback. At higher feedback levels, the availability of multiple solutions could lead to frequency hopping or splitting. If the phase of the feedback light is scanned back and forth, a frequency hysteresis can be observed. This behavior is known as Regimes II and III. Regime IV corresponds to the laser diode entering coherence collapse while, finally, stable external cavity operation starts at high feedback levels in Regime V. While classification in regimes serves as a good basis for comparison, it is important to note that more complicated devices, such as DBR tapered laser diodes, might behave differently due to the lateral mode dynamics, small internal reflections from transitions between internal sections of the device or other variations from a simpler single transverse mode device.

For low levels of feedback, the linewidth will either increase or decrease, and the center frequency will shift, depending on both the phase and strength of the feedback. However, the linewidth change is small and can be difficult to observe. For this reason, we focus on the shift of the frequency, which can be on the order of a few hundred MHz. A simple laser with two mirrors, perturbed by feedback from a single external source, will undergo a frequency shift. The frequency shift for low feedback powers can be approximated by the theory given in [11]

$$
\Delta \omega \equiv \omega-\omega_{0}=-\kappa\left(\sin \left(\omega \tau_{e}\right)+\alpha \cos \left(\omega \tau_{e}\right)\right)
$$

where $\omega$ is the frequency, $\omega_{0}$ is the frequency without feedback, $\tau_{e}$ is the round trip time for the external feedback, $\alpha$ is the linewidth enhancement factor and $\kappa$ is defined by: 


$$
\kappa=\frac{1}{\tau_{s}} \frac{1-R_{s}}{\sqrt{R_{s}}} \sqrt{R_{e}}
$$

It depends on the internal round trip time of the laser, $\tau_{\mathrm{s}}$, the power reflectivity of the output coupler, $R_{\mathrm{s}}$ and the power reflectivity of the external feedback source, $R_{\mathrm{e}}$, with total attenuation, $\mu_{\mathrm{AT}}$, in the feedback branch given by

$$
\mu_{\mathrm{AT}}=10 \log _{10} R_{\mathrm{e}}
$$

It should be noted that both $R_{\mathrm{e}}$ and $R_{\mathrm{S}}$ represent the ratios of the power that are mode-matched back into the resonator. Therefore, the value of $R_{\mathrm{s}}$ will not be identical to the power reflection of the front facet coating on neither a DFB laser nor a tapered laser diode.

Of the five regimes, the border between Regime I and II is especially interesting since it describes the maximum feedback that the laser diode can handle before the frequency shift becomes discontinuous, which, according to the theory, should occur when Acket's factor $C$, exceeds one [19].

$$
C=\sqrt{1+\alpha^{2}} \kappa \tau_{e}>1
$$

This discontinuity in the frequency shift could lead to further instabilities and therefore impact laser-based experiments or applications [20]. For this reason, the transition from regime I to II is measured and used as a quantification of how sensitive the laser diodes are to optical feedback as this will severely impact the stability of a frequency-converted laser system.

Despite some of the lasers used in this work have more complicated geometries, the comparison of the experimental results with the simulations based on the theory in [11] is a good starting point to understand the influence of external feedback on the devices.

\section{The experimental setup}

The feedback power ratios of interest to this paper extend down below $-100 \mathrm{~dB}$. Such low values are very challenging to obtain in a reproducible manner.

The main consideration for the setup is how to ensure a high coupling efficiency for the intended feedback while eliminating the feedback from other elements in the setup. For this reason, the feedback branches were designed to have as few and as simple optical elements as possible, and the diagnostics branch of the setup is isolated using a Faraday isolator. A schematic of the setup is shown in Figure 1. The plane optics are slightly angled with respect to the optical axis to ensure that reflections are not coupled back into the laser diode. To stabilize the coupling efficiency of the feedback, the light is focused onto a mirror as this makes the coupling efficiency relatively independent of the mirror alignment.

We use an uncoated wedge as a beam pick-off for the controlled feedback. Since the laser diodes used are p-polarized relative to the wedge surface, the Fresnel reflectivity is approximately $1 \%(-20 \mathrm{~dB})$, so the maximum amount of feedback in the configurations described in this section is approximately $-40 \mathrm{~dB}$. If an s-polarized laser is used instead, the Fresnel reflectivity is approximately $10 \%$, resulting in a maximum of approximately $-20 \mathrm{~dB}$. If even higher levels of feedback are needed, then the positions of the diagnostics and the feedback branches can be switched since the diagnostics equipment requires very little light. The main focus of this work is feedback in regimes I and II. Therefore, the configuration with a maximum feedback power ratio of $-40 \mathrm{~dB}$ was chosen.

Before any measurement is performed, it is important to ensure that no component in the setup is producing too much unwanted feedback. This can be difficult to do in practice, but here we utilize the fact that the effect of low levels of feedback is highly dependent on the phase of the feedback. This means that if a component is giving significant feedback, then the emission frequency will be shifted if the component is moved along the optical axis by half a 
wavelength of the light. In practice, this can be done by lightly touching the mount of the optics. The emission frequency change can easily be in the hundreds of $\mathrm{MHz}$ and is therefore visible on the scanning Fabry Perot interferometers.

\subsection{Setup}

Figure 1 shows a schematic of the setup. The laser diode output is collimated in the fast axis using an aspherical lens. For the tapered laser, a cylindrical lens is used to collimate the beam in the slow axis to correct for the astigmatism. To investigate whether the collimation optics influenced the results either by different mode matching or different amounts of unwanted feedback, we tested two different lens combinations for the tapered laser. The focal length of the aspherical lens is either $2 \mathrm{~mm}$ or $4.5 \mathrm{~mm}$. The cylindrical lenses used for collimating the tapered laser have focal lengths of $5.8 \mathrm{~mm}$ or $25.8 \mathrm{~mm}$, respectively. After the laser diode is collimated, an uncoated wedge is used to select a portion of the light for the controlled feedback.

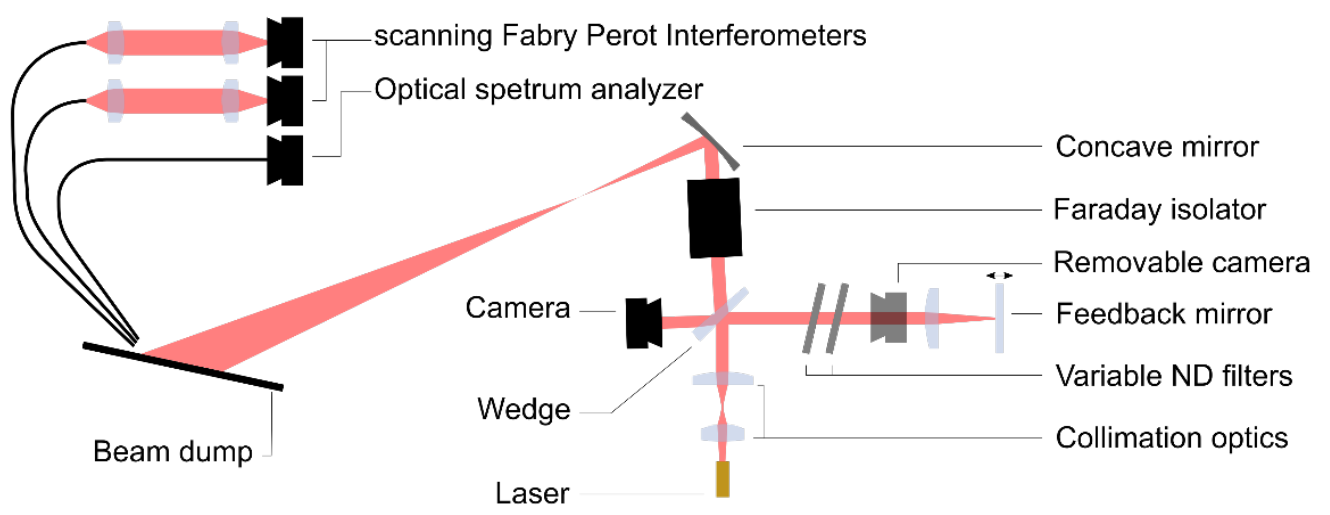

Figure 1. Schematic of the setup. The fast axis of the laser beam is collimated using a biconvex aspheric lens. If the laser diode is a tapered laser diode, then a cylindrical lens is added to collimate the laser beam in the slow axis as well due to astigmatism.

This light passes two reflective neutral density (ND) filters. The light is then focused onto a mirror using a lens with a focal length of $45 \mathrm{~mm}$. The mirror is mounted on a piezoelectric transducer, and both the mirror and the focusing lens in the feedback arm are mounted on a rail so that the distance to the laser diode can be varied from $25 \mathrm{~cm}$ to $100 \mathrm{~cm}$. The feedback power ratio is measured by inserting a power meter just before the feedback mirror and comparing the power here with the known output power of the laser. As the light travels back to the laser diode with the same attenuation, the ratio includes both passes of the attenuators. The light transmitted through the wedge is passed through a $30 \mathrm{~dB}$ isolator and expanded with a concave mirror onto a beam dump. For diagnostics, two scanning Fabry Perot interferometers (sFPI, Thorlabs SA200-8B, and SA210-8B) are used together with an optical spectrum analyzer (OSA, Advantest Q8347). To avoid unwanted feedback from the diagnostics equipment, we use multi-mode fibers to collect scattered light from the laser diode and the beam dump for the OSA and sFPIs, respectively. To ensure proper collimation of the laser diode, a camera and a lens can be inserted after the ND filters to image the beam waist. The feedback beam is monitored with a second camera. This camera is also used in the alignment process.

\subsection{Unwanted feedback}

The unwanted reflections from the cylindrical lens, the wedge, the ND filters, and the focusing lens can be avoided by tilting the elements and dumping the reflections, so that they will not 
be coupled back into the laser diode. However, this leaves two components from where the unwanted reflections can be unavoidable.

The aspherical lens used for fast-axis collimation cannot be angled, due to the high numerical aperture needed. To calculate the amount of feedback from this element, we used the ray-tracing software BeamXpertDESIGNER to estimate the amount of light reflected into the entrance to the ridge waveguide of a tapered laser diode. For the $f=2 \mathrm{~mm}$ lens, the estimated feedback is $-79 \mathrm{~dB}$ and $-77 \mathrm{~dB}$ from the lens' first and second interface, respectively, assuming a $0.2 \%$ AR coating. A similar estimate for the $f=4.5 \mathrm{~mm}$ lens in the alternative optics yields $-80 \mathrm{~dB}$ and $-88 \mathrm{~dB}$ feedback from the lens' first and second interface, respectively, with the same coating. Unfortunately, this level of feedback can be enough to measurably affect sensitive laser diodes. We have done calculations on other types of collimation optics, including aspherical lenses with a longer focal length. For longer focal lengths, the radius of curvature of the lens is higher, giving a less divergent reflection, so no significant improvement is observed in theory or practice. Most other collimation schemes use a combination of cylindrical lenses where the plane side is towards the laser, which increases the level of feedback. The sensitivity to feedback in regime I increases with the distance to the external reflection, and for this reason, it is difficult to estimate how much the feedback from the fast axis collimator will affect the results.

The other component that may cause problems is the Faraday isolator itself. Firstly, it gives rise to many extra reflections that need to be dumped, and especially the light that is rejected by the two polarizing beam splitters (PBS) can cause problems if dumped on surfaces that are not sufficiently absorbing at the relevant wavelength. Therefore, it is paramount that the isolator used allows these beams to exit the isolator, as the internal beam dump surfaces are typically not absorbing sufficiently. On top of this, the scattering from the coating inside the first PBS and bulk scattering in the crystal used for the Faraday rotation can be sufficient to have an impact on the measurements on sensitive laser diodes. For the work presented here, the measurements were only performed on lasers where it was ensured that the feedback from these components was in regime I.

\subsection{Alignment}

Common for both lens combinations is the need for aligning the feedback mirror to achieve a high coupling efficiency. This needs to be done not only when a new laser diode is inserted in the setup but also every time the feedback mirror is moved to a new position as the setups currently have no way to monitor whether the coupling efficiency has changed. This alignment can be done in two fundamental ways.

The first option is to minimize the threshold for the laser. This is done by operating the laser close to threshold and measure the emitted power while applying a high amount of feedback. Improving the coupling efficiency will lower the threshold of the laser and lead to a higher emitted power. This alignment is by far the easiest method, but for the tapered laser diodes, it has the drawback that the alignment of the cylindrical lens depends on the astigmatism, which in turn depends on the emitted power. For this reason, at least one camera is needed to ensure the beam does not move during a subsequent realignment of the cylindrical lens.

The second way to align the feedback mirror for high coupling efficiency is to monitor the signal from the sFPI while adjusting the feedback mirror and maximizing the feedback signature. Due to the large instabilities at higher levels of feedback, this alignment should be performed either in or close to regime I feedback. In this regime, the amplitude of the wavelength shift depends on the feedback power ratio, and this amplitude can, therefore, be maximized. This type of alignment has the advantage that it can be performed at the same operating parameters for the laser as will be used for the measurements, but it is timeconsuming and requires some training before it can be done in a way that yields reproducible 
results. Furthermore, it requires continuous scanning of the feedback mirror during the alignment to observe the phase dependence of the feedback.

We tested both alignment procedures against each other by integrating a beam waist camera and found that they gave the same results with respect to the alignment of the feedback mirror. The threshold alignment procedure was therefore used when a camera was integrated into the setup since this alignment can be done significantly faster.

\section{Experimental results}

The setup was tested first at low optical powers $(<150 \mathrm{~mW})$ with a DFB laser. We performed this initial test to compare the setup to the results in the literature. After verification, the setup was used to analyze the emission behavior under small external feedback of high power DBR tapered lasers.

\subsection{DFB laser}

The DFB laser used for characterization of both setups was produced at the Ferdinand-BraunInstitut. It has a length of $1.5 \mathrm{~mm}$, a ridge waveguide (RW) width of $3 \mu \mathrm{m}$, a waveguide thickness of $3.6 \mu \mathrm{m}$, and a front facet AR coating with $R_{\mathrm{f}}<0.5 \%$. For all shown measurements, we applied an injection current of $200 \mathrm{~mA}$ to the laser and temperature stabilized it to a heat sink temperature of $25^{\circ} \mathrm{C}$. At these drive conditions, the laser emits at $975 \mathrm{~nm}$ with a spectral width of less than $32 \mathrm{fm}(10 \mathrm{MHz})$.

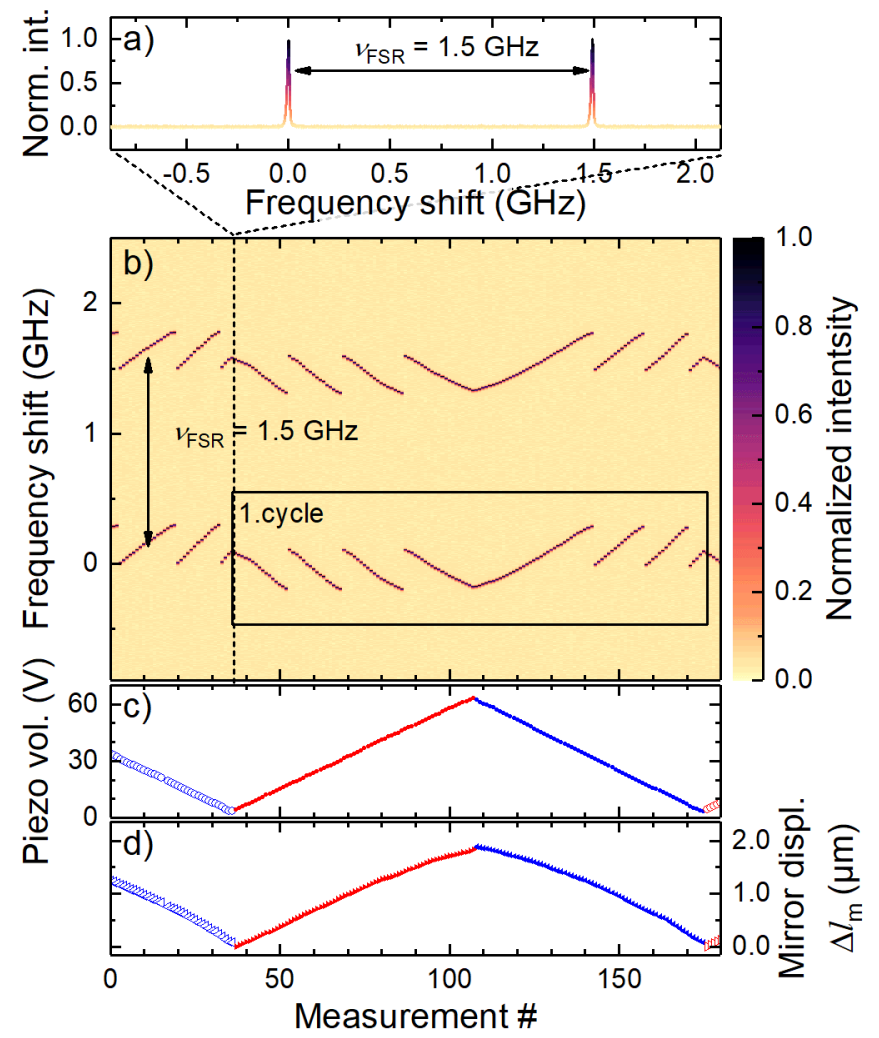

Figure 2. Illustration of the measurement concept. a) A single Fabry Perot interferometer measurement, showing a cross-section of the false-color plot in b). Example b) frequency spectra, c) piezo voltage, and d) mirror displacement for the DFB laser at $\mu_{\mathrm{AT}}=-45 \mathrm{~dB}$ feedback, measured with a distance to the center position of the mirror of $l_{\mathrm{e}}=350 \mathrm{~mm}$. 
A triangular voltage function was used to scan the piezo-mounted feedback mirror and thereby change the external cavity length $l_{\mathrm{e}}=350 \mathrm{~mm}+\Delta l_{\mathrm{m}}$. In Figure $2(\mathrm{~b})$, the frequency spectra measured with the scanning Fabry Perot interferometer are shown in a false-color plot for 180 measurement samples. The free spectral range $v_{\mathrm{FSR}}=1.5 \mathrm{GHz}$ of the sFPI is highlighted in the graph, and one full cycle of the mirror movement is shown. A single measured spectrum is shown in Figure 2 (a), showing the false-color scale used in Figure 2 (b). The single measured spectrum, as well as the false-color plot, are normalized by the maximum measured intensity of the complete measurement set. The acquisition rate of the frequency spectra was not constant and was not synchronized with the scan rate of the piezo. From the simultaneously measured piezo voltage, shown in (c), a direct correlation between mirror position and measured frequency spectra can be derived based on the hysteresis data provided by the distributor of the piezo actuator, as can be seen in (d).

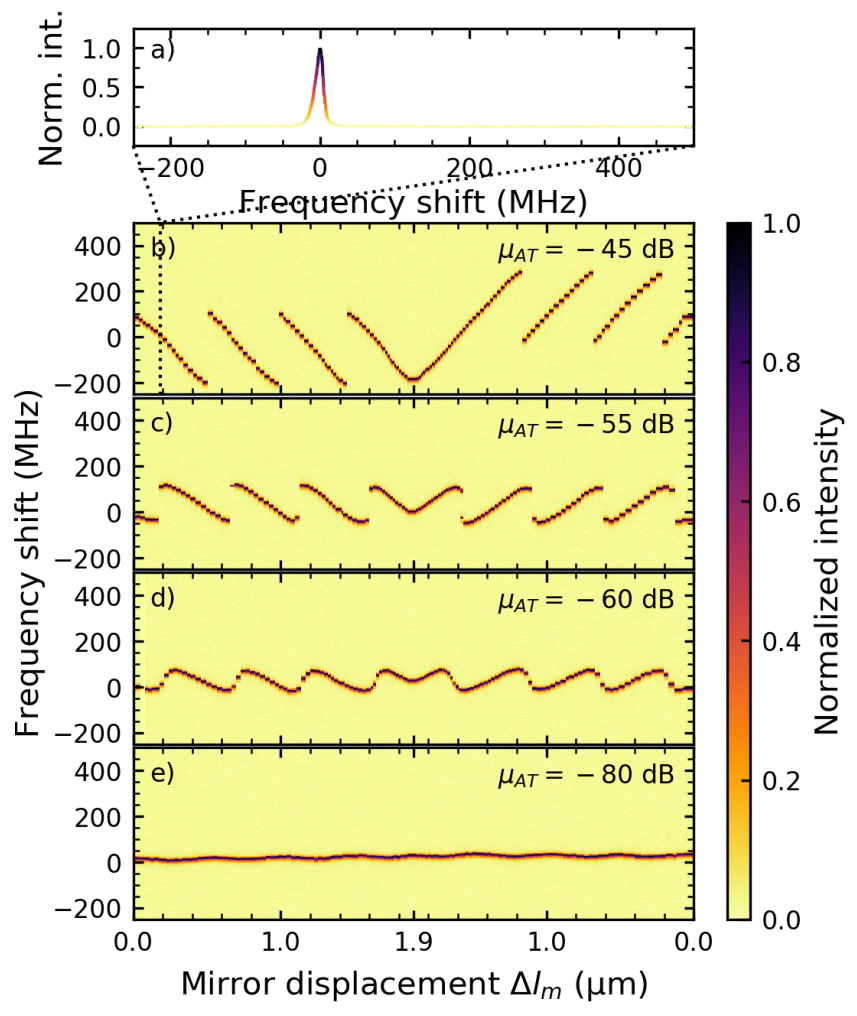

Figure 3. Frequency shift for one cycle of piezo mounted mirror movement for the DFB laser at various feedback power ratios $\mu_{\mathrm{AT}}$. The distance to the center position of the mirror was $350 \mathrm{~mm}$. The feedback mirror was moved $1.9 \mu \mathrm{m}$ towards the laser and back. The inset (a) shows a Fabry Perot interferometer measurement from a single measurement.

In Figure 3, the frequency shift for one cycle of mirror movement is shown in a false-color plot for the DFB laser at different feedback power ratios $\mu_{\mathrm{AT}}$. At $\mu_{\mathrm{AT}}=-80 \mathrm{~dB}$ (Figure 3 (e)) the influence of the external feedback on the emission frequency is not resolvable with this setup, limited by its stability. With increasing feedback power ratio, the amplitude of the frequency shift increases. The frequency shift is nonlinear but continuous with the change of the length of the external feedback cavity, as can be seen in (d) at a feedback power ratio of $\mu_{\mathrm{AT}}=-60 \mathrm{~dB}$. Between $\mu_{\mathrm{AT}}=-60 \mathrm{~dB}$ and $\mu_{\mathrm{AT}}=-55 \mathrm{~dB}$ the frequency shift becomes discontinuous when the feedback mirror position is changed, as can be seen in (c). For a feedback power ratio of $\mu_{A T}=-45 \mathrm{~dB}$, this discontinuity is still present, and the frequency is 
seen in (b) to exhibit hysteresis with regards to the direction in which the mirror is moved. The width of the frequency envelope stays the same at approximately $300 \mathrm{MHz}$. For all measured feedback power ratios, the emission is single frequency.

This is all in good agreement with the theory, as can be seen in Figure 4. In this graph, the frequency shift is plotted as a function of the mirror position. The two different translation directions of the feedback mirror are indicated by the color of the measurement points. If the mirror is moved towards the laser, the measurement points are blue. If it is moved away from the laser, the measurement points are colored red. The solid black line is the calculated frequency shift, according to [11]. The following parameters were used for the simulation:

$$
\begin{gathered}
\tau_{\mathrm{s}}=34 \mathrm{ps} \\
R_{\mathrm{s}}=5 \%(\text { fitted }) \\
\tau_{\mathrm{e}}=2.3 \mathrm{~ns} \\
R_{\mathrm{e}}=10^{\left(\mu_{A T} / 10\right)} \\
\alpha=2
\end{gathered}
$$

The internal round trip time $\tau_{\mathrm{s}}$ and the external feedback cavity round trip time $\tau_{\mathrm{e}}$ depend on the length of the laser with $l_{\mathrm{s}}=1.5 \mathrm{~mm}$ and the external resonator length $l_{\mathrm{e}}=350 \mathrm{~mm}$. The reflectivity of the external feedback $R_{\mathrm{e}}$ is based on the measured values and the linewidth enhancement factor of $\alpha=2 \pm 0.1$, which was measured following the method in [21]. The effective power reflectivity of the output coupler $R_{\mathrm{s}}=5 \%$ was fitted to the measured data of the maximum frequency shift. The reflectivity of the output coupler depends on the front facet reflectivity and the reflectivity of the DFB grating. The front facet of the DFB laser has an AR coating with $R_{\mathrm{f}}<0.5 \%$. In combination with the reflectivity of the DFB grating, a value of $R_{\mathrm{S}}=5 \%$ is realistic.

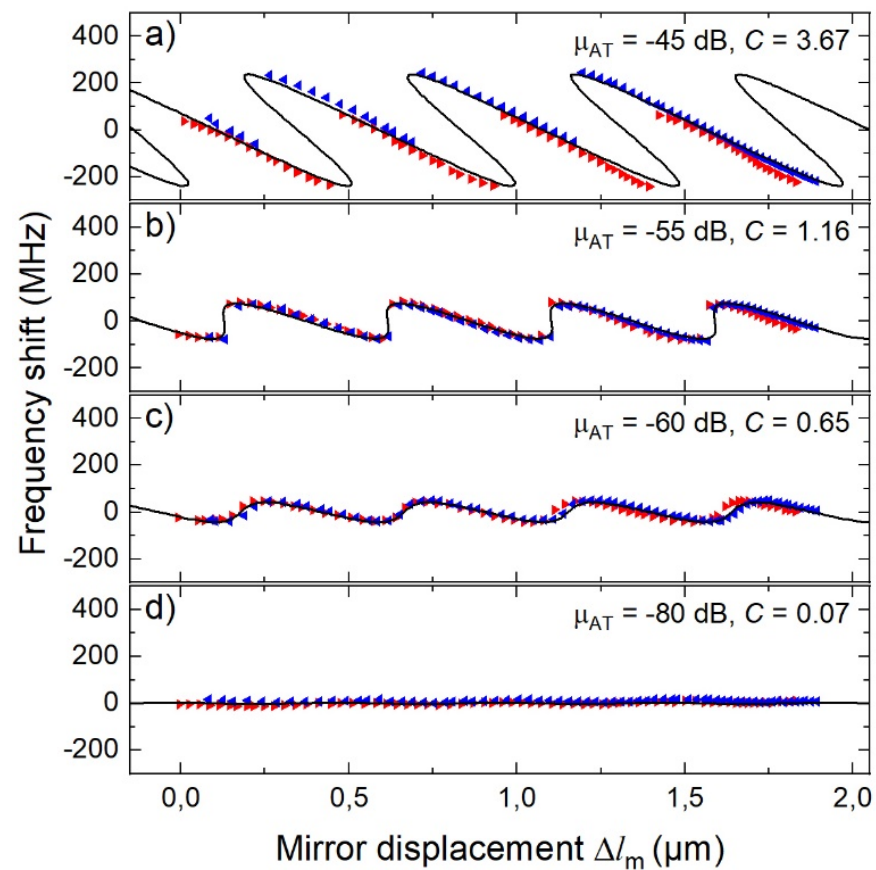

Figure 4. Frequency shift vs. mirror displacement for the DFB laser at various feedback power ratios $\mu_{\mathrm{AT}}$. The distance to the center position of the mirror was $350 \mathrm{~mm}$. The measurement for a positive and negative mirror movement direction are shown in red and blue, respectively. The solid line shows the simulated frequency alteration. $C$ is Acket's factor, defined in (4) [19]. 


\subsection{DBR tapered laser}

The same measurements were performed with a DBR-TPL. This laser consists of three sections, starting at the backside with a DBR section, followed by a RW section and a tapered amplifier (TA) section. The DBR section is a $1 \mathrm{~mm}$ long, tapered $7^{\text {th }}$ order DBR grating with a design reflectivity of $95 \%$. The RW section has a length of $1.5 \mathrm{~mm}$ and a width of $5 \mu \mathrm{m}$. The gain guided tapered section is $3.5 \mathrm{~mm}$ long with a full taper angle of $6^{\circ}$ and a front facet coating of $R_{\mathrm{f}}=0.3 \%$. The waveguide thickness is $4.8 \mu \mathrm{m}$

For all following measurements, we applied the injection currents $I_{\mathrm{RW}}=490 \mathrm{~mA}$ and $I_{\mathrm{TA}}=4500 \mathrm{~mA}$ to the RW and TA sections, respectively. At these drive conditions, the laser emits $2 \mathrm{~W}$ of optical power at $1066 \mathrm{~nm}$ with a spectral width of less than $57 \mathrm{fm}(15 \mathrm{MHz})$. The power contained in the central peak is greater than $85 \%$. The laser was temperature stabilized to a heat sink temperature of $25^{\circ} \mathrm{C}$.

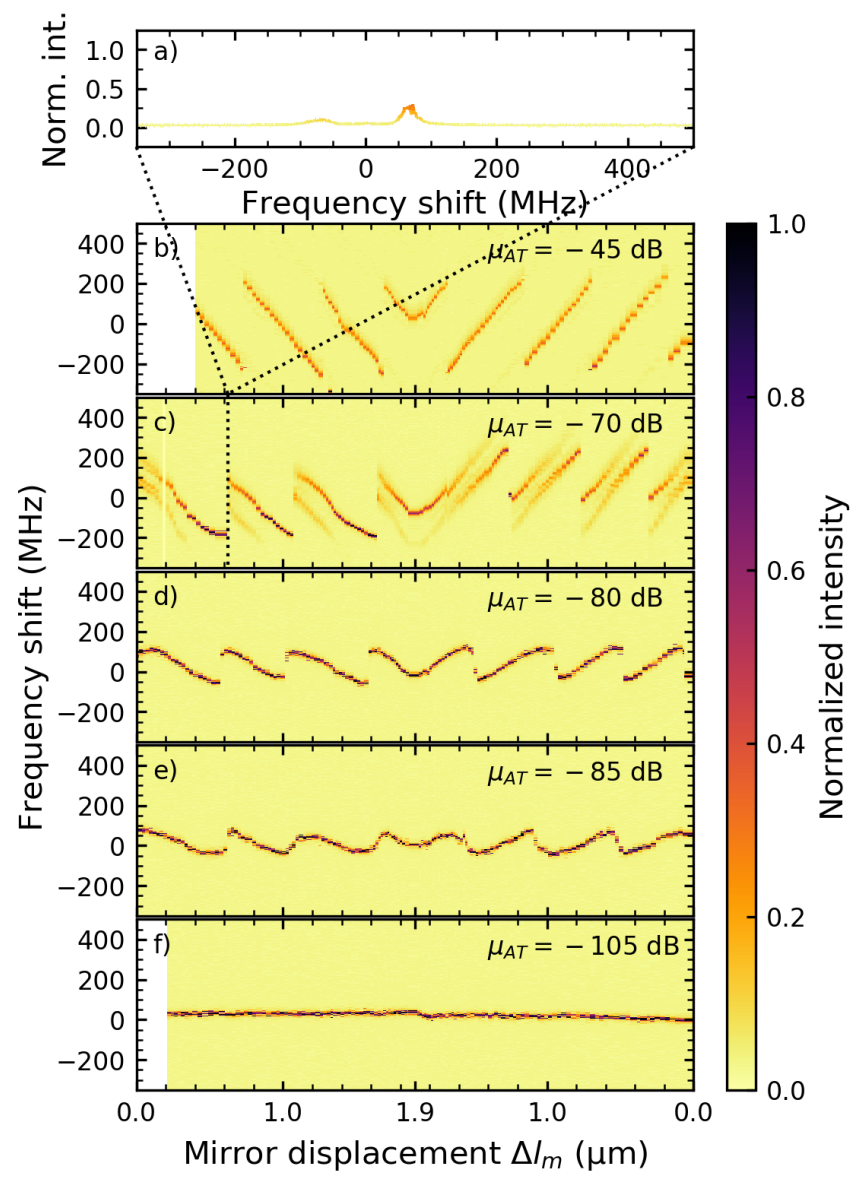

Figure 5. Frequency shift for one cycle of piezo mounted mirror movement for the DBR-TPL at various feedback power ratios $\mu_{\mathrm{AT}}$. The distance to the center position of the mirror was $350 \mathrm{~mm}$. The feedback mirror was moved $1.9 \mu \mathrm{m}$ towards the laser and back. The inset (a) shows a Fabry Perot interferometer measurement from a single measurement.

In Figure 5, the frequency shift for one cycle of mirror movement is shown in a false-color plot for several levels of feedback power ratio $\mu_{\mathrm{AT}}$. The feedback power ratio at which no influence by external feedback can be measured is $\mu_{\mathrm{AT}}=-105 \mathrm{~dB}$, shown in (f). If the feedback power ratio is increased, the frequency shift is first continuous with the mirror movement (e), 
and then becomes discontinuous at feedback levels just above the one shown in (d). The feedback sensitivity is orders of magnitude greater for this laser compared to the DFB laser because tapered lasers are designed with a much lower effective front facet reflectivity $R_{\mathrm{s}}$ due to the very high amplification in the tapered section. This low $R_{\mathrm{s}}$ is the result of both a lowreflectivity coating on the front facet and the fact that the light reflected back from the front facet is diverging and thus not well mode-matched into the ridge section.

The transition from a continuous to a discontinuous frequency shift in dependence of the mirror movement occurs at a feedback power ratio slightly higher than $-80 \mathrm{~dB}$. For higher feedback power ratios, the emission can become multi-frequency, as can be seen for $\mu_{\mathrm{AT}}=-70 \mathrm{~dB}$ in Figure 5 (c) and (a). At this feedback power ratio, the laser emits at two frequencies. Both the frequencies and the power distribution between them depend on the feedback mirror position, but the spacing between both frequencies is independent of the mirror position. If the feedback power ratio is increased further, single-frequency emission can again occur, as can be seen in (b). One may speculate that diode lasers operating at high powers, such as the tapered lasers studied here, are subject to a wealth of different types of nonlinear effects including filamentation and thermal effects that, together with the complex behavior of the time-delayed optical feedback, can cause more types of instability than is included in the theoretical model used in this work. The multi-frequency operation seen in (c) may be such an instability.

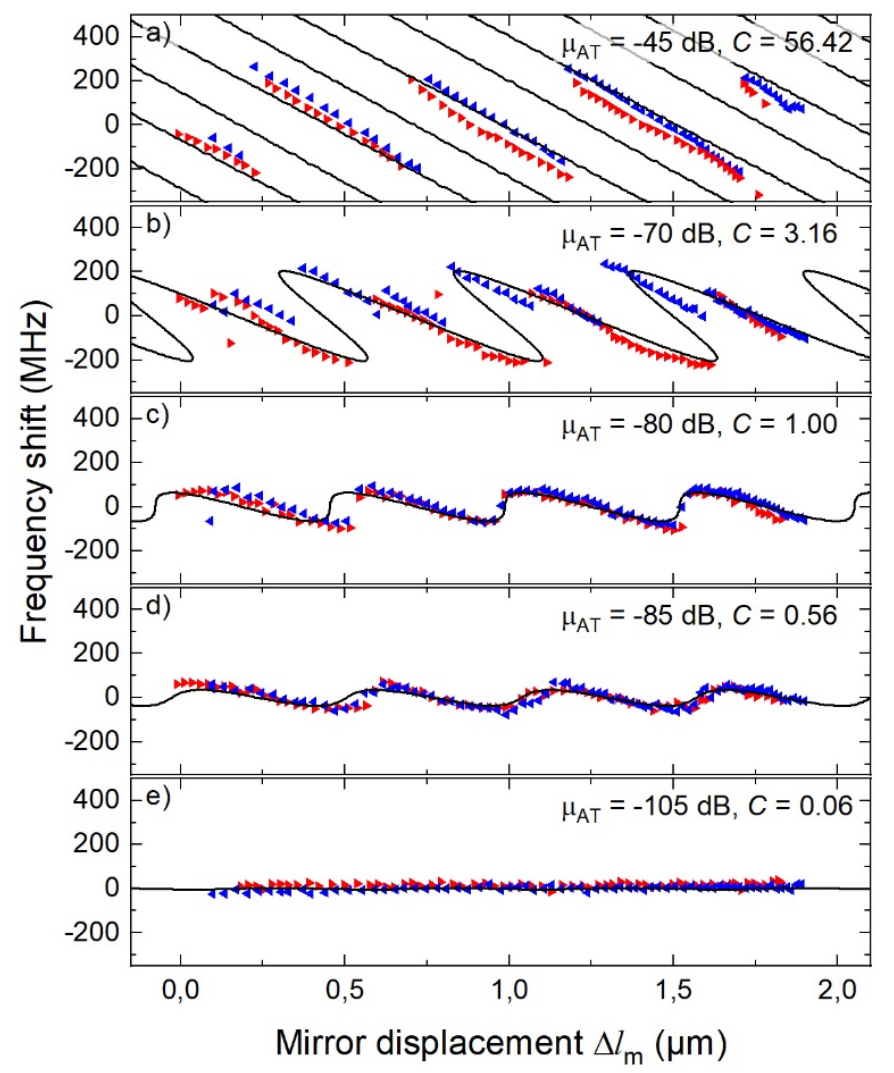

Figure 6. Frequency shift vs. mirror displacement for the DBR-TPL laser at various feedback power ratios $\mu_{\mathrm{AT}}$. The measurement for a positive and negative mirror movement direction are shown in red and blue, respectively. The solid line shows the simulated frequency shift. The distance to the mirror was $350 \mathrm{~mm}$. $C$ is Acket's factor, defined in (4) [19]. 
In Figure 6, the center frequency of the peak with the highest intensity is plotted as a function of the mirror position (red and blue triangles) and compared with the simulated data (solid black line). The following parameters were used to calculate the simulated data:

$$
\begin{gathered}
\tau_{\mathrm{s}}=125 \mathrm{ps} \\
R_{\mathrm{s}}=0.0013 \% \text { (fitted) } \\
\tau_{\mathrm{e}}=2.3 \mathrm{~ns} \\
R_{\mathrm{e}}=10^{\left(\mu_{\mathrm{AT}} / 10\right)} \\
\alpha=1.5
\end{gathered}
$$

The linewidth enhancement factor for the DBR tapered laser was $\alpha=1.5 \pm 0.1$, measured following the method in [21] and is comparable to published values for similar material $[22,23]$. The time constants are based on the effective internal resonator length of $l_{\mathrm{s}}=5.5 \mathrm{~mm}$ and the mean external cavity length $l_{\mathrm{e}}=350 \mathrm{~mm}$. The fitted value for the output coupler reflectivity is $R_{\mathrm{S}}=0.0013 \%$. This value is in the same order of magnitude as the calculated effective power reflectivity of the output coupler,

$$
R_{\mathrm{s} \text { eff }}=R_{\mathrm{f}} C_{\text {top-hat }}=0.0009 \% \text {, }
$$

consisting of the product of the power reflectivity of the front facet coating of $R_{\mathrm{f}}=0.3 \%$ and the correction factor $C_{\text {top-hat }}$ based on the estimate of the additional loss of re-seeding the RW with the diverging back-reflected top-hat beam coming from the tapered section [24].

$$
C_{\text {top-hat }} \approx \frac{n w_{\mathrm{RW}}^{2}}{2 \lambda L_{\mathrm{TA}}}=0.003,
$$

where $n=3.4$ is the effective refractive index, $w_{\mathrm{RW}}=2.5 \mu \mathrm{m}$ is the horizontal beam waist radius at $1 / \mathrm{e}^{2}, \lambda=1066 \mathrm{~nm}$ is the wavelength, and $L_{\mathrm{TA}}=3.5 \mathrm{~mm}$ is the length of the tapered section. At low feedback power ratios, the measured data is in good agreement with the simulated data. Even at a feedback power ratio of $\mu_{\mathrm{AT}}=-70 \mathrm{~dB}$, where multi-frequency emission occurs, the range of the frequency shift and the width of the mode jump free mirror movement can be approximated following [11]. At $\mu_{\mathrm{AT}}=-45 \mathrm{~dB}$ only the slope of the frequency alteration versus the mirror position and the frequency spacing of the mode jumps are in good agreement with the theory.

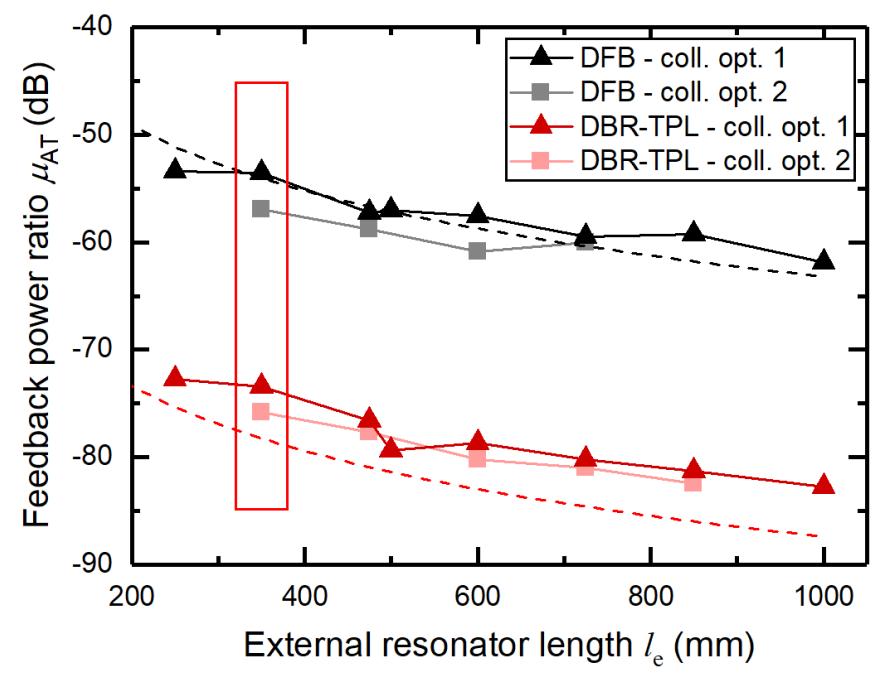

Figure 7. Measured regime borders I-II as a function of the external resonator length for both laser diodes and collimation optics. The red frame indicates the external cavity length for the measurements shown in Figure 2-6. The dashed lines show the calculated border between regimes I and II.

In addition to the analysis of the frequency shift, we also measured the border between 
regimes I and II. In regime I, the frequency alteration is continuous with the mirror displacement, while in regime II, the shift becomes discontinuous, and mode jumps occur. The regime border is dependent on the external cavity length, as can be seen in Figure 7 in agreement with [11]. Included in Figure 7 are theoretical curves indicating the border between regime I and II. Highlighted in the red frame is the external cavity length for the measurements shown in Figure 2-6. Good agreement between measurements with two different collimation optics and the theory is evident for both laser diodes. However, a small offset is seen for the measurements on the DBR tapered laser. This may be the result of a slightly inferior modematching of the feedback light and a higher frequency noise compared to the DFB laser.

\section{Summary}

In this paper, we presented a detailed description of a measurement setup and method to analyze the influence of external feedback on high-power single-frequency laser diodes. We used a low power DFB laser to characterize the setup and test the measurement method. The results are in good agreement with the theory based on the Lang-Kobayashi equations and with results in the literature. Furthermore, we showed that this measurement method is also suitable for high-power laser diodes, as shown for DBR-TPLs. At low feedback power ratios, the results are in good agreement with the theory. At high feedback power ratios, only certain parameters such as the frequency spacing of the mode jumps and the slope of the frequency shift in dependence of the mirror displacement are in agreement with the theory.

The proposed experimental techniques allow a quantitative assessment and classification of the effects of external feedback on high power laser diodes. This may lead to better parameter selection and design of laser diodes.

\section{Funding and disclosures}

\subsection{Funding}

This work was supported by the European Commission within the projects MIB 667933-2 and FBI 721766, and by the Innovation Fund Denmark under grant number 5016-00076B

\subsection{Disclosures}

The authors declare no conflicts of interest.

\section{References}

1. B. Sumpf, K.-H. Hasler, P. Adamiec, F. Bugge, J. Fricke, P. Ressel, H. Wenzel, G. Erbert, and G. Tränkle, "1060 nm DBR tapered lasers with $12 \mathrm{~W}$ output power and a nearly diffraction limited beam quality," in Proc. SPIE, A. A. Belyanin and P. M. Smowton, eds. (2009), Vol. 7230, pp. 72301E-72301E-8.

2. C. Fiebig, G. Blume, C. Kaspari, D. Feise, J. Fricke, M. Matalla, W. John, H. Wenzel, K. Paschke, and G. Erbert, "12 W high-brightness single-frequency DBR tapered diode laser," Electron. Lett. 44, 1253-1255 (2008).

3. A. Müller, J. Fricke, F. Bugge, O. Brox, G. Erbert, and B. Sumpf, "DBR tapered diode laser with $12.7 \mathrm{~W}$ output power and nearly diffraction-limited, narrowband emission at $1030 \mathrm{~nm}, "$ Appl. Phys. B 122, 87 (2016).

4. A. K. Hansen, M. Tawfieq, O. B. Jensen, P. E. Andersen, B. Sumpf, G. Erbert, and P. M. Petersen, "Concept for power scaling second harmonic generation using a cascade of nonlinear crystals," Opt. Express 23, 15921-15934 (2015).

5. R. S. Hansen and C. Pedersen, "All semiconductor laser Doppler anemometer at $1.55 \mu \mathrm{m}, "$ Opt. Express 16, 18288-18295 (2008).

6. B. Tromborg, J. Osmundsen, and H. Olesen, "Stability analysis for a semiconductor laser in an external cavity," IEEE J. Quantum Electron. 20, 1023-1032 (1984).

7. M. W. Fleming and A. Mooradian, "Spectral Characteristics of External-Cavity 
Controlled Semiconductor Lasers," IEEE J. Quantum Electron. 17, 44-59 (1981).

8. K. Petermann, "Exernal Optical Feedback Phenomena in Semiconductor Lasers," IEEE J. Sel. Top. Quantum Electron. 1, 480-489 (1995).

9. T. Kurosaki, T. Hirono, and M. Fukuda, "Suppression of External Cavity Modes in DFB Lasers with a High Endurance against Optical Feedback," IEEE Photonics Technol. Lett. 6, 900-902 (1994).

10. M. Vilera, M. Christensen, A. K. Hansen, P. Arulthasan, D. Noordegraaf, T. Buß, O. B. Jensen, P. E. Andersen, and P. M. W. Skovgaard, "2.7 W diffraction-limited yellow lasers by efficient frequency doubling of high-brightness tapered diode lasers," Opt. Commun. 435, 145-149 (2019).

11. R. W. Tkach and A. R. Chraplyvy, "Regimes of feedback effects in $1.5-\mu \mathrm{m}$ distributed feedback lasers," J. Light. Technol. LT-4, 1655-1661 (1986).

12. S. Donati and R. Horng, "The Diagram of Feedback Regimes Revisited," IEEE J. Sel. Top. Quantum Electron. 19, 1500309 (2013).

13. S. Donati and M. T. Fathi, "Transition From Short-to-Long Cavity and from SelfMixing to Chaos in a Delayed Optical Feedback Laser," IEEE J. Quantum Electron. 48, 1352-1359 (2012).

14. S. Donati, "Developing self-mixing interferometry for instrumentation and measurements," Laser Photonics Rev. 6, 393-417 (2012).

15. S.-S. Li, V. Pusino, S.-C. Chan, and M. Sorel, "Experimental investigation on feedback insensitivity in semiconductor ring lasers," Opt. Lett. 43, 1974-1977 (2018).

16. D. Jedrzejczyk, P. Asbahr, M. Pulka, B. Eppich, and K. Paschke, "High-Power Single-Mode Fiber Coupling of a Laterally Tapered Single-Frequency Diode Laser," IEEE Photonics Technol. Lett. 26, 845-847 (2014).

17. R. Bege, D. Jedrzejczyk, G. Blume, J. Fricke, F. Bugge, K. Paschke, and G. Tränkle, "Reduction of Optical Feedback Originating From Ferroelectric Domains of Periodically Poled Crystals," IEEE J. Quantum Electron. 53, 9000109 (2017).

18. R. Lang and K. Kobayashi, "External optical feedback effects on semiconductor injection laser properties," IEEE J. Quantum Electron. 16, 347-355 (1980).

19. G. A. Acket, D. Lenstra, A. J. D. Boef, and B. H. Verbeek, "The Influence of Feedback Intensity on Longitudinal Mode Properties and Optical Noise in IndexGuided Semiconductor Lasers," IEEE J. Quantum Electron. 20, 1163-1169 (1984).

20. A. K. Hansen, M. Christensen, D. Noordegraaf, P. Heist, E. Papastathopoulos, O. B. Jensen, and P. M. W. Skovgaard, "Efficient Generation of $1.9 \mathrm{~W}$ yellow light by Cascaded Frequency Doubling of a DBR Tapered Diode Laser," Appl. Opt. 55, 9270-9274 (2016).

21. Y. Yu, G. Giuliani, and S. Donati, "Measurement of the linewidth enhancement factor of semiconductor lasers based on the optical feedback self-mixing effect," IEEE Photonics Technol. Lett. 16, 990-992 (2004).

22. D. V. Batrak, S. A. Bogatova, A. V. Borodaenko, A. E. Drakin, and A. P. Bogatov, "Simulation of the material gain in quantum-well InGaAs layers used in 1.06- $\mu \mathrm{m}$ heterolasers," Quantum Electron. 35, 316-322 (2005).

23. J. Stohs, D. J. Bossert, D. J. Gallant, and S. R. J. Brueck, "Gain, refractive index change, and linewidth enhancement factor in broad-area GaAs and InGaAs quantum-well lasers," IEEE J. Quantum Electron. 37, 1449-1459 (2001).

24. S. Delepine, F. Gerard, A. Pinquier, T. Fillion, J. Pasquier, D. Locatelli, J.-P. Chardon, H. K. Bissessur, N. Bouche, F. R. Boubal, and P. Salet, "How to launch 1 $\mathrm{W}$ into single-mode fiber from a single $1.48-\mu \mathrm{m}$ flared resonator," IEEE J. Sel. Top. Quantum Electron. 7, 111-123 (2001). 